\title{
National inpatient diagnostic hysteroscopy survey
}

\author{
Sherif Tawfeek Peter Scott
}

Received: 21 June 2009 / Accepted: 9 July 2009 /Published online: 28 July 2009

(C) Springer-Verlag 2009

\begin{abstract}
This survey was undertaken to determine the common practise patterns of how hysteroscopy is carried out in the UK. We believe this will help to develop a consensus and set national guidelines in accordance with the way we undertake this procedure. In addition; we aim to assess the operators understanding of hysteroscope optics related to this procedure. The study was conducted in a teaching hospital in the southwest of England, UK. Postal questionnaires were sent within the first week of June 2007 to 1,000 consultant gynaecologists in the UK. The surveyed gynaecologists were selected from the Royal college of Obstetricians and Gynaecologists (RCOG) register of Fellows and Members. The questionnaire included questions regarding common preoperative preparation, operative techniques, post-operative management, and follow-up. Four hundred seventy-five $(47.5 \%)$ responses were returned. Four hundred forty (44\%) consultant gynaecologists completed the survey and 35 (3.5\%) incomplete questionnaires were returned by consultants who currently do not undertake hysteroscopy. It appears that there is some consensus in with the way we undertake this procedure. However, the results showed a wide variation in the understanding of the hysteroscopes angled optics and how this relates to surgical technique for inpatient hysteroscopy. It seems that setting up a national guideline is feasible. However, there was a clear lack of understanding of the optics used during a hysteroscopic procedure between
\end{abstract}

\footnotetext{
S. Tawfeek $(\bowtie)$

Obstetrics and Gynaecology, Southmead Hospital,

Bristol, UK

e-mail: sherif@tawfeek.freeserve.co.uk

P. Scott

Obstetrics and Gynaecology, Derriford Hospital,

Plymouth, UK
}

gynaecologists. Optics-related surgical techniques should be included into training programmes. Professional societies are able to develop a general consensus for this procedure. We are pleased that the practical skills courses run by the RCOG include clear training in this area.

Keywords Hysteroscopy · Diagnostic · Inpatient · Survey · Optic

\section{Introduction}

Diagnostic hysteroscopy (DH) is considered one of the most frequent gynaecological procedures [1-3]. It has become an effective method for diagnosing of various intra-uterine pathologies [4]. Like all endoscopic procedures, it requires thorough training, practical experience and familiarity with the endoscopic techniques [5]. Awareness of possible complications and precautions to prevent them is also important [6].

We therefore conducted a national questionnaire survey of consultant gynaecologists in the UK. The intended goal was to find if a consensus was feasible. Also, it was to assess the operators' understanding of the optics of the hysteroscope.

\section{Material and methods}

A self-administered questionnaire to all 1,000 UK consultant gynaecologists to enquire about their current practise and potential willingness to participate in a randomised trial.

A two-page questionnaire was developed for this study (Appendix 1). It composed 30 questions. The questionnaire 
was a structured format consisting of a series of closed, opened and multiple choice questions. However, the participant was able to add his/her preferred answer if it was different with the given choices. In addition, some questions require the participant to provide numbers e.g. hysteroscope size etc. Each surveyed envelope contained a letter of introduction, the questionnaire and a self-addressed return envelope.

After a pilot testing on 50 of the surveyed participants, the questionnaire was sent to all gynaecologists $(n=1,000)$. The anonymous nature of the survey makes comparisons of those who did and did not respond to the survey impossible. Therefore, there was no reminder sent for those who failed to respond. This survey was not funded by any industry source.

The first question in our survey has addressed the potential operative risks. Questions 2-24 were focused on the intra-operative preparation and individual techniques. However, question 19 was estimating the participants' understanding of the optics of hysteroscopy through choosing one of the supplied three photos showing different positions of the intra-cervical canal. We have linked questions 11 and 19 in recording the correct answer for question 19 as the correct answer depends on the degree of angulations of the scope lens. Question 25 was to find the participants' preference on follow-up. Also, we tried to find in question 26 the participant's percentage of inpatientoutpatient hysteroscopy. Questions 27-29 were to identify the participant experience in using and availability of operative hysteroscopy. The last question asked for any comment the participant would add.

The received information was collected in the statistical SPSS programme (SPSS, version 11, SPSS, Chicago, IL, USA).

\section{Results}

Of the postal mail sent, $475(47.5 \%)$ were returned and of them $440(44 \%)$ were completed questionnaires. Thirty-six (3.5\%) consultant gynaecologists who do not perform hysteroscopies, returned their questionnaire incomplete. This includes obstetricians, or retired consultants. The results of the completed questionnaire were entered into the computer database. Not all responders answered every question; hence, there are discrepancies between the total number of returned questionnaires and the total number reported for some questions.

In response to the question of preoperative informed consent; 34, 49, 58 and $147(n=423)$ respondents did not consent their patients for uterine perforation, bleeding, infection and anaesthetic complication, respectively.

General anaesthesia was chosen most commonly as the preferred anaesthetic method by $58 \%$ of respondents among other types. Anaesthetist preference is the second commonly recorded answer. The majority of respondents neither catheterise $(92 \%)$ nor give antibiotics $(98 \%)$. More than two-thirds of those surveyed reported that they prefer lithotomy position to Lloyd-Davies position. Examination under anaesthesia is routinely used by $(89.5 \%)$. Of the respondents, $85.5 \%$ do not have an assistant in theatre and $57 \%$ check their instruments themselves. Cuscos speculum is less likely used $(7.6 \%)$ compared to Sims speculum (78.6\%). However, $13.8 \%$ of respondents do not use any speculums at all. The majority of respondents reported that cervical dilatation depends on the scope size and the cervix is always checked at the end of the procedure.

Over two-thirds, $65.8 \%$, of those surveyed start the fluid media at the cervix, $30.5 \%$ start the fluid media prior to scope entry and $3.7 \%$ start the fluid media within the uterine cavity. However, most try to empty the uterine cavity at the end. The majority do not use any fluid suction system. Table 1 shows the surveyed gynaecologists preference regarding scope size, scope degree, type of fluid media and fluid pressure, respectively. Advancing the scope under camera vision was selected as the most commonly used technique.

The response to question 19 was high; 397. Of respondents, $66.6 \%$ using $0^{\circ}$ scope chose the correct photo. Only $25.8 \%$ of respondents using $25-70^{\circ}$ scope chose the correct photo. The overall correct answer for this question was $38.8 \%$.

The common (61.6\%) employed assessment system for the uterus starts with the uterine fundus, followed by tubal ostia, uterine wall and ends by examining the cervical canal. Two-thirds of responders attempted taking an endometrial biopsy. Table 2 shows the operators' preference to record the findings.

The response to question 27, 28 and 29 showed that the resectoscope is available in theatre in $80.5 \%$ of the respondents; however, only $69.5 \%$ have been trained and are able to use it.

Table 1 Surveyed gynaecologists preference

\begin{tabular}{llr}
\hline Scope size & $5 \mathrm{~mm}$ & 176 \\
& $3 \mathrm{~mm}$ & 58 \\
Scope degree & $30^{\circ}$ & 169 \\
& $0^{\circ}$ & 124 \\
Fluid media & $\mathrm{N}$. saline & 388 \\
& $\mathrm{CO}_{2}$ & 26 \\
Fluid pressure & $40-60 \mathrm{mmHg}$ & 21 \\
& $80-100 \mathrm{mmHg}$ & 122 \\
& $100-150 \mathrm{mmHg}$ & 101 \\
& Manual & 61 \\
& No pressure & 44 \\
\hline
\end{tabular}


Table 2 Operators' preference

\begin{tabular}{lc}
\hline Ways to record the operative findings & No. \\
\hline Photos only & 96 \\
Photos and hand written records only & 89 \\
Hand written records only & 81 \\
Photos, diagrams and hand written records & 61 \\
\hline
\end{tabular}

\section{Discussion}

This survey was conducted to observe a difference in the current UK practise patterns in undertaking diagnostic hysteroscopy. It also assesses the operators understanding of the hysteroscope optics.

Diagnostic hysteroscopy may be performed without anaesthesia [7]. Although local anaesthetics, intravenous sedation and paracervical blocks may be adequate for diagnostic hysteroscopy, they may not be suitable for more prolonged or complicated procedure [8].

Dilatation of the cervix and introduction of the hysteroscope are to blame for the risk of uterine perforation. Introduction of the hysteroscope under direct vision reduces this risk [6]. On other hand, dilatation of the cervix for diagnostic procedures is not mandatory [9-11]; however, others dilated the cervix routinely during all diagnostic procedures without complications [12].

The position, size and shape of the uterus should be assessed by bimanual examination if cervical dilatation is indicated [5]. Bladder emptying is not a routine unless it interferes with bimanual uterine assessment. The smaller the scope size the less likely dilatation required, 3-5 mm scope is recommended [13].

'No-touch' procedure of vaginoscopy was pioneered by Bettocchi and Salvaggi in 1995 [14]. It was performed with 5-mm diameter, continuous flow hysteroscope without medication, cervical dilatation or the use of vaginal speculum or cervical tenaculum. Initially, the labia minora may have to be held together to facilitate distention of the vagina during the vaginoscopy part. The uterine distention is using saline at a pressure of approximately $30 \mathrm{mmHg}$. The failure rates of vaginoscopic procedures have ranged from 0 to $2.4 \%$ [14-16].

Cervical priming has been considered pre-operatively especially with expected difficult cervical dilatation. The use of misoprostol (400 $\mu \mathrm{g}$ orally or $200 \mu \mathrm{g}$ vaginal) 9 to $12 \mathrm{~h}$ prior to the surgery, was shown to be beneficial in both pre-menopausal and post-menopausal women [17].

The mean intra-uterine pressure required to separate the uterine walls is $40 \mathrm{mmHg}$ (range 25-50 mmHg) [18] and spill from normal fallopian tubes occurs at $70 \mathrm{mmHg}$ when saline is the distending medium [19]. There is no ideal medium, and no one medium is devoid of complications. Comparing normal saline with $\mathrm{CO}_{2}$ as distending media resulted in similar pain during the procedure with less postoperative discomfort or bowel irritation with normal saline [20]. In addition, $\mathrm{CO}_{2}$ costs substantially more than normal saline [21].

Preventing hysteroscopic complications start by raising the understanding of the hysteroscope optics which was one of our main objectives in this study. Some of the survey's questions are designed to check the understanding of the hysteroscope optics through choosing the correct photo when the angled or $0^{\circ}$ scope is used. Only $25.8 \%$ of respondents chose the correct photo which we believe is low.

About half of the complications caused by the hysteroscopic approach were related to entry of the uterine cavity [6]. Therefore, advancing the hysteroscope under direct vision is recommended [6].

In order to know that the telescope and cervical canal are directly in line prior to advancing, the view on the screen needs to be fully understood to minimise the risk of uterine perforation and also to minimise pain experienced by patients in the outpatient setting.
Fig. 1 Expected correct pictures with angled or $0^{\circ}$ scope

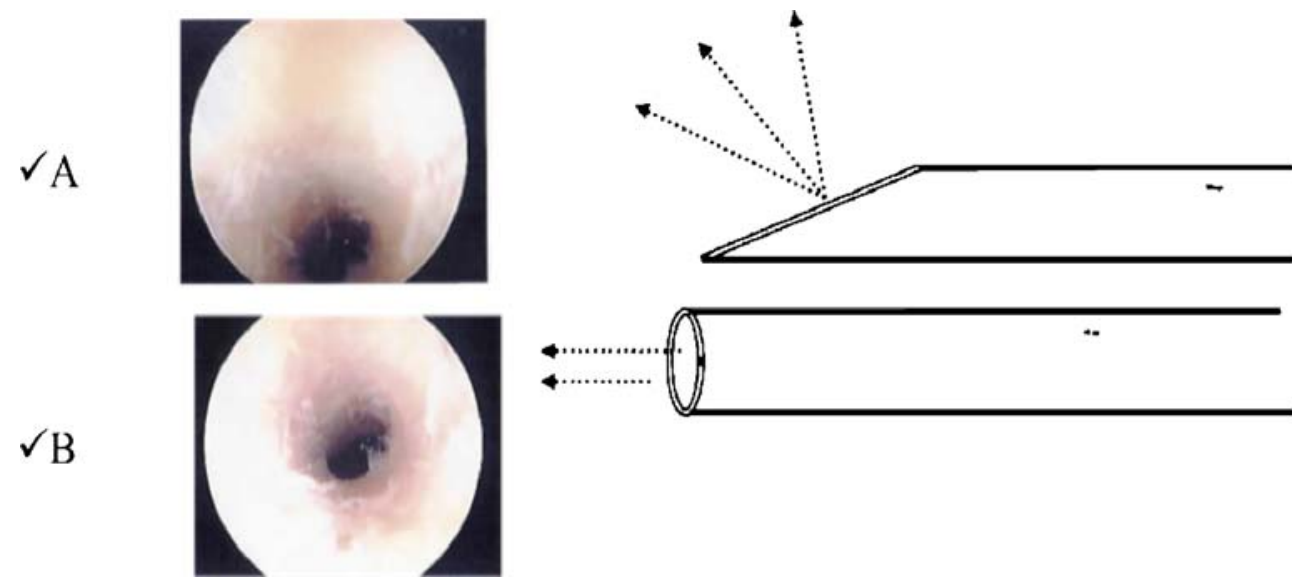




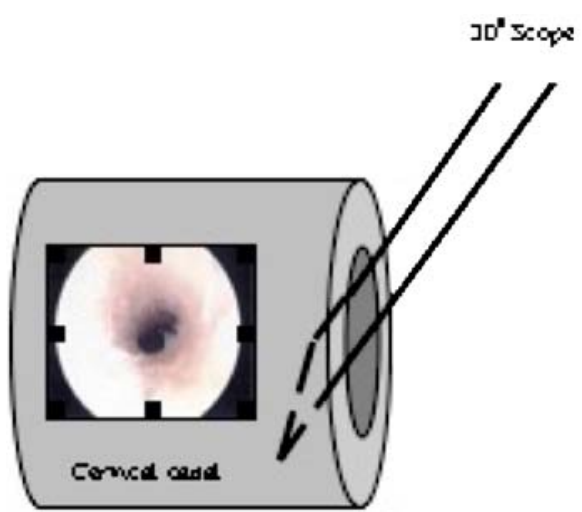

Fig. 2 The telescope is angled posteriorly with a significant risk of a posterior perforation when the $30^{\circ}$ scope is advanced

With a $30^{\circ}$ angled scope the scope and canal are only inline when you can just see the cervical canal at the bottom of the picture with angle scope looking upwards. With $0^{\circ}$ scope, you will see the cervical canal in the middle of the picture (Fig. 1). If the cervical canal is in the middle of the picture then the telescope is angled posteriorly with a significant risk of a posterior perforation when the scope is advanced (Fig. 2). Vice versa with the angled scope looking down, requires the canal to be at the 12 o'clock position for the scope on cervical canal to be inline.
It is disappointing that only $25.8 \%$ of responders answered this question correctly and indicates that at the consultant level this is poorly understood and therefore poorly taught to future generations.

We are pleased that this subject is taught clearly and correctly in the Royal college of Obstetricians and Gynaecologists practical skills course. We are hopeful and confident that future generations of gynaecologists will not perpetuate those misunderstandings.

\section{Conclusion}

There was a lack of understanding of the optics of the hysteroscopic procedure between gynaecologists. We strongly recommended that the optics-related surgical techniques should be included into any hysteroscopic training programme. Professional societies are able to develop a general consensus for this procedure. This would improve the understanding of hysteroscopy, reduce complications and aid setting up a guideline for the procedure.

Conflict of interest There is no actual or potential conflict of interest in relation to this article. 


\title{
Appendix
}

\section{National Inpatient Diagnostic Hyteroscopy survey}

1. What complications do you inform patients of?

Uterine perforation

Bleeding

Infection

Anaesthetic risks

Others: (please specify).......

2. What type of anaesthesia do you prefer? Nil

\author{
Spinal \\ General \\ Local \\ Anaesthetist preference
}

3. Do you catheterise patients pre-operatively? Yes No

4. Do you use antibiotics at induction? $\quad$ Yes No

5. How do you position the patients?

Lithotomy
Lloyd-Davies position
Others (please specify)...........

6. Do you examine your patient in theatre under anaesthesia prior to the procedure?

$\begin{array}{lllll}\text { 7. Do you request an assistant (staff nurse or doctor)? } & \text { Yes } & \text { No } & \text { Usually } \\ & \text { No } & \text { Usually }\end{array}$

8. Do you usually check and assemble instruments yourself pre-operatively Yes No

9. Do you use a speculum during hysteroscopy? Yes No

If yes Type: Sims

Cusco's

Others:.......

10. What size/diameter hysteroscope do you prefer? . $\mathrm{mm}$

No preference

Unknown

11. What degree of scope lens do you use $\quad \begin{array}{llll}0^{0} & 25^{0} & 30^{\circ} & 70^{\circ}\end{array}$

No preference

Unknown

12. How far do you dilate the cervix to? Nil

Depend on the hyteroscope size

Others:

13. What distension medium do you prefer? Normal saline Glycine $\mathrm{CO} 2$

Water

Sorbitol

Other (please specify):......

14. When do you start the fluid medium flow?
A. Prior to entry
B.In the cervical canal
C. In the uterine cavity 
15. Do you use a fluid suction system?

16. What pressure do you prefer:
Yes

No

No pressure $80-100 \mathrm{mmHg}$ cuff Manual pressure
$100-150 \mathrm{mmHg}$ cuff $40-60 \mathrm{mmHg}$ cuff

Unknown

17. Do you use a camera or direct eye view?

18. Do you advance the hyteroscope under direct vision? Yes

No

19. Where do you position the cervical canal on the monitor screen prior to advancing the scope you chose previously above if performed under direct vision with the light lead down?

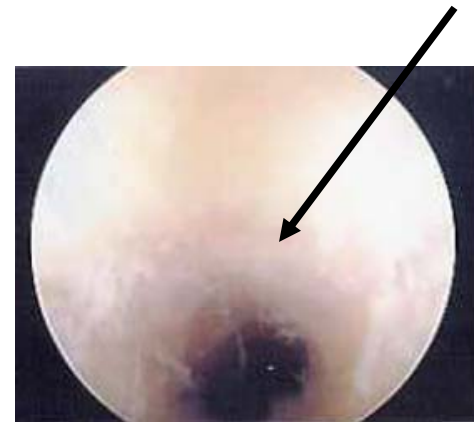

1

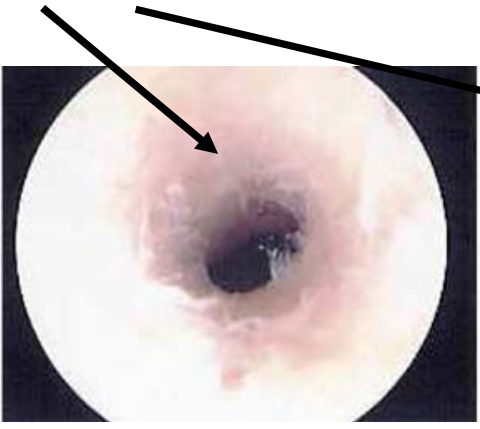

2

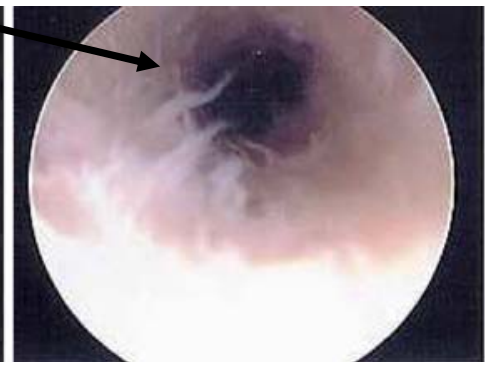

3

20. What is preferred assessment system for the uterus? (Start with the first)

Fundus Tubal ostia Uterine wall Cervical canal.

21. Do you attempt to take endometrial biopsy even if the uterine cavity looks normal?

Yes No

22. Do you usually try to empty the residual fluid from uterine cavity at exit? Yes No

23. How do you prefer to record the findings?

Photos if available

Video if available

Diagrams

Hand written record

Others:

24. Do you check the cervix at the end of surgery? Yes

No

25. How do you follow up your patients?

A.Review before discharge B.Gynaecology follow-up clinic C.Write back with the histology result

26. What is your percentage of hysteroscopy: Inpatient $\%$ : Outpatient $\%$

27. Is the resectoscope available in theatre if required? Yes

No

28. Are you able to use the resectoscope if required? Yes

No

29. Have you been trained to use resectoscope?

Yes

No

30. Other comments: (Please use overleaf if required) 


\section{References}

1. Serden SP (2000) Diagnostic hysteroscopy to evaluate the cause of abnormal uterine bleeding. Obstet Gynecol Clin North Am 27:277-286

2. Nagele F, O'Connor H, Davies A et al (1996) 2500 outpatient diagnostic hysteroscopies. Obstet Gynecol 88:87-92

3. Unfried G, Wieser F, Albrecht A et al (2001) Flexible versus rigid endoscopes for outpatient hysteroscopy: a prospective randomized clinical trial. Hum Reprod 16:168-171

4. Gimpelson R, Rappold H (1998) A comparative study between panoramic hysteroscopy with directed biopsies and dilatation and curetage: a review of 276 cases. Am J Obstet Gynecol 158:489-492

5. Vilos GA, Abu-Rafea B (2005) New developments in ambulatory hysteroscopic surgery. Best Pract Res Clin Obstet Gynaecol 19:727-742

6. Jansen FW, Vredevoogd CB, Ulzen KV et al (2000) Complications of hysteroscopy: a prospective, multicenter study. Obstet Gynecol 96:266-270

7. Siegler J (1995) Office hysteroscopy. Obstet Gynecol Clin N Amer 22:457

8. Ananthanarayan C, Paek W, Rolbin SH et al (1996) Hysteroscopy and anaethesia. Can J Anaesth 43:56-64

9. Jong P, Doel F, Falconer A (1990) Outpatient diagnostic hysterocopy. Br J Obstet Gynaecol 97:299-303

10. Baggish MS (1997) Operative hysteroscopy. In: Rock CA, Thompson JD (eds) Te Linde's operative gynaecology, 8th edn. Lippincott-Raven, Philadelphia, pp 415-442

11. Wamsteker K (1997) Endoscopy: hysteroscopy. In: Brosens I, Wamsteker K (eds) Diagnostic imaging and endoscopy in gynecology; a practical guide. Saunders, London, pp 17-38
12. Cutner A, Erian J (1996) Who should have outpatient hysteroscopy? Gynaecol Endosc 5:231-234

13. Bakour SH, Jones SE, O'Donovan P (2006) Ambulatory hysteroscopy: evidence-based guide to diagnosis and therapy. Best Pract Res Clin Obstet Gynaecol 20:953-975

14. Bettocchi S, Selvaggi L (1997) A vaginoscopic approach to reduce the pain of office hysteroscopy. J Am Assoc Gynecol Laparosc 4:255-258

15. Bettocchi S, Ceci O, Di Venere R et al (2002) Advanced operative office hysteroscopy without anaesthesia: analysis of 501 cases treated with a 5 Fr. bipolar electrode. Hum Reprod 17: 2435-2438

16. Carluccio MG, Marello F, Caliandio D et al (2004) Office hysteroscopy: pain evaluation by visual analog scale. J Am Assoc Gynecol Laparosc 11:S54

17. Preutthipan S, Herabutya Y (2000) Vaginal misoprostol for cervical priming before operative hysteroscopy: a randomized controlled trial. Obstet Gynecol 96:890-894

18. Baker VL, Adamson GD (1998) Minimum intrauterine pressure required for uterine distention. J Am Assoc Gynecol Laparosc $5: 51-53$

19. Baker VL, Adamson GD (1995) Threshold intrauterine perfusion pressures for intraperitoneal spill during hydrotubation and correlation with tubal adhesive disease. Fertil Steril 64:10661069

20. Pellicano M, Guida M, Zullo F et al (2003) Carbon dioxide versus normal saline as a uterine distension medium for diagnostic vaginoscopic hysteroscopy in infertile patients: a prospective, randomized, multicenter study. Fertil Steril 79:418-421

21. Brusco GF, Arena S, Angelini A (2003) Use of carbon dioxide versus normal saline for diagnostic hysteroscopy. Fertil Steril 79:993-997 